\section{LA MEJORA EN LA MORTALIDAD POR CIRUGÍA DE URGENCIA EN PACIENTES MAYORES REQUIERE ATENCIÓN SOBRE EL RESCATE DE LAS COMPLICACIONES. Sheetz KH. Ann Surg. 2013;258:614-8.}

Es sabido que la mortalidad quirúrgica aumenta exponencialmente con la edad. Estimaciones conservadoras muestran que alrededor de un tercio de la población mayor experimenta alguna cirugía en el último año de su vida. En los Estados Unidos la población mayor de 65 años hoy representa el $12 \%$ de la población, pero va a crecer al $20 \%$ en el año 2030 , este hecho representa un desafío mayor. Evidencia reciente muestra una mortalidad hasta 10 veces mayor en cirugía de urgencia en pacientes añosos cuando se la compara con una población más joven. Si bien se ha fomentado la aplicación de las mejores prácticas para mitigar el riesgo perioperatorio, no está claro qué es lo mejor para reducir la mortalidad: o prevenir las complicaciones o tratarlas de la mejor forma. Algunos impugnan la baja reserva fisiológica de los pacientes mayores como la causa de la mayor morbilidad y mortalidad; otros, en cambio, plantean que hay diferencias en los distintos hospitales, debido a las distintas estructuras y sistemas de cuidado.

La importancia de estos factores no se ha estudiado en el ambiente de cirugía de urgencia. Por esta razón, los autores evaluaron entre los años 2006 y 2011 lo que sucedió en 41 hospitales de Michigan Surgical Quality Collaborative, observando la evolución de 23.224 pacientes sometidos a cirugía de urgencia general o vascular, comparando pacientes por bajo y por sobre los 75 años de edad. Se estudió el riesgo ajustado de complicaciones mayores y la falla para rescatarlos de la complicación (failures- to-rescue), traducido como mortalidad post complicación mayor. Se separaron los hospitales en tres terciles según su mortalidad.

Luego de hacer los ajustes por factores clínicos, diagnóstico y volumen hospitalario la mortalidad promedio en los hospitales según terciles fue de $6,4 \%, 7,4 \%$ y $8,9 \%$. La morbilidad, en cambio, no varió significativamente y fluctuó entre $25,6 \%$, $21,8 \%$ y $25,9 \%$. Al comparar la mortalidad por sobre los 75 años, esta fue de $17,5 \%, 19,6 \%$ y $22,8 \%$ en cada grupo de hospitales. La tasa de complicaciones mayores no se correlacionó con la mortalidad. Pero, la incapacidad de los hospitales para rescatar y sacar adelante a los pacientes complicados fue marcadamente distinta entre hospitales de baja y de alta mortalidad. Esto último se observó en población joven y mayor. Para los hospitales de alta mortalidad, la falla en el rescate se observó en el $42,1 \%$ en casos de alta mortalidad y en el $27,4 \%$ en los de baja mortalidad.

Los datos expuestos permiten notar que una falla en el rescate de los pacientes complicados está en la base de una mayor mortalidad, más que la tasa de complicaciones propiamente tal. Aunque la baja reserva fisiológica de los pacientes mayores lleva a una baja en el rescate y eso explica en parte la mortalidad, la amplia variación entre hospitales, hace pensar que son altamente importantes los sistemas que ayudan a reconocer precozmente las complicaciones y la implementación de medidas efectivas de manejo, especialmente en esta población vulnerable.

Dr. Ricardo Espinoza G. Universidad de los Andes Santiago, Chile 